\title{
Spectral Iterative Algorithm for RCS Computation in Electrically Large or Intermediate Perfectly Conducting Cavities
}

\author{
Juan M. Rius, Member, IEEE, Angel Lozano, Lluis Jofre, Member, IEEE, and Angel Cardama, Member, IEEE
}

\begin{abstract}
A novel algorithm designed to compute efficiently and accurately the high-frequency electromagnetic scattering from open-ended waveguide cavities is presented. The cavity is converted into a stepped-waveguide model so that the field spectra are propagated, forward and backward, along each waveguide section. As boundary conditions for perfect electric conductors are applied via image theory, they are of local nature and take into account only the first-order interactions between each pair of waveguide sections. Accordingly, additional forward-backward iterations must be performed if multiple interactions are to be taken into account. Finally, the radar cross section due to the interior irradiation is calculated by a Kirchhoff-based aperture integral. Good agreement with Method of Moments and Hybrid Modal solutions is found, as well as with experimental data, for two-dimensional and three-dimensional cavities with rectangular cross section.
\end{abstract}

\section{INTRODUCTION}

$\mathbf{T}$ HE analysis of electromagnetic (EM) scattering from open-ended waveguide cavities has received strong attention in the last years in relation to radar cross section (RCS) reduction and target signatures. For RCS analysis of complex targets, duct structures such as jet engine intakes can often be modeled by more simple waveguide cavities.

When the dimensions are electrically small, numerical techniques such as Method of Moments (MoM) [1] or Finite Elements Method (FEM) [2] can be applied, leading to rigorous solutions. In electrically large cavities, on the other hand, high frequency (HF) approximations must be used to compute the interior radiation due to the large number of unknowns involved in the numerical solution. In cavities with regular uniform geometry, modal methods have proved to be efficient over a broad range of frequencies [3], [4], though the geometrical constraints prevent realistic modeling of complex targets.

In the case of more arbitrarily shaped cavities, geometrical optics based ray-shooting [5] or Gaussian beam (GB) [6] approaches can be used to deal with smooth nonuniform geometries, but they are limited to very high frequency problems. Recently, several hybrid schemes [7], [8] have been reported to treat cavities that can be modeled by uniform waveguide sections connected by arbitrary transitions, though

Manuscript received April 19, 1993; revised December 6, 1993. This work was supported under Grant CICYT-TIC-93-S18.

The authors are with the Antennas, Microwave and Radar Group, Dpt. Teoria del Senyal i Comunicacions, Universitat Politécnica de Catalunya, 08080 Barcelona, Spain.

IEEE Log Number 9402811. some of them [8] introduce low-frequency techniques in the $\mathrm{HF}$ approximations, which results in an important increase in the computational requirements.

Another relevant scheme reported recently [9] allows for the low-frequency analysis of three-dimensional cavities using a connection technique closely related to the Microwave Network Theory. It is based on the division of the cavity into sections which are independently analyzed. When each of them has been represented by a generalized admittance matrix, the aperture admittance is derived by cascading the matrixes of every individual section.

We present in this paper an alternative technique conceptually related to the above-mentioned connection scheme [9]. Our technique is based on the spectral domain representation of electromagnetic fields, first introduced by Bojarski in the 1970's [15], [16]; it can be considered an evolution of previously existing spectral methods, namely the Spectral-Iterative Technique (SIT) [10] and the Spectral Incremental Procedure (SIP) [11]. The new technique provides the approximate RCS of simple cavity structures in a broad frequency range with high efficiency, and with a progressive refinement of the solution. This particular feature adapts the computational cost to the complexity of the cavity.

The algorithm, however, accounts only for the interior irradiation, so that asymptotic methods such as Physical Theory of Diffraction (PTD) [12], Method of Equivalent Currents (MEC) [13], or Uniform Theory of Diffraction (UTD) [14] should be employed to include first-order edge effects. If the cavity is embedded in a more complex body, classical theories such as physical optics (PO) could be used to incorporate the external scattering.

The formulation of the algorithm will begin with the description of the two-dimensional version, which is conceptually more simple. Extension to a 3-D nonuniform rectangular cross section will follow. Results for both two-dimensional and three-dimensional cavities will be presented to illustrate the performance of the method and to establish comparison with reported results. Finally, the features and drawbacks of the algorithm will be discussed.

\section{TWo-DIMENSIONAL ALgoRITHM}

An arbitrary 2-D cavity is plotted in Fig. 1. To begin with, a segmentation process must be carried out in order to turn the original geometry into a stepped-waveguide cavity 


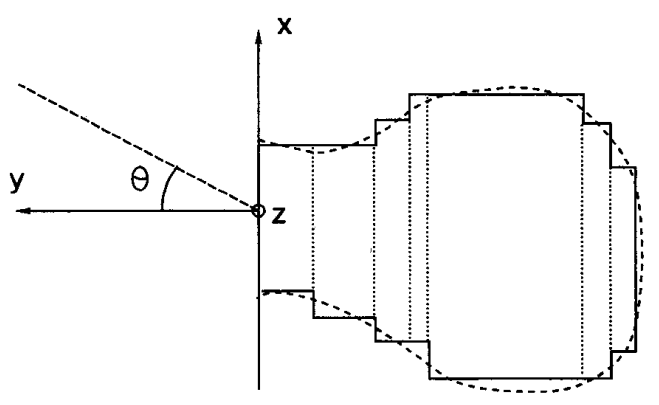

Fig. 1. Segmentation process in an arbitrary 2-D cavity. Dotted line: Original cavity. Solid line: Stepped-waveguide model.

consisting of several straight sections, each corresponding to a parallel plate waveguide. The resolution required in the stepped model will be discussed later, but it depends mainly on geometrical complexity and smoothness of the original cavity, computational resources, and requested accuracy.

Once the modeling is completed, incident fields due to a plane-wave excitement are found via Kirchhoff's Approximation over the aperture. These fields are then spectrally propagated successively through every waveguide section, where local boundary conditions are applied. Forward radiation of equivalent currents is obtained directly via image theory, while backward radiation is stored to be used later in the process. Once the end-plate of the cavity is reached and the forward propagation procedure is thus finished, a backward propagation must follow. Reflected fields previously stored are now added to the propagating wavefront at every waveguide section, before local boundary conditions are applied. Forward reflected fields must still be stored if further iterations are to be carried out. The outwardly traveling fields obtained over the aperture can be considered as a first-order approximation for the interior scattering. As it is well known that HF diffraction is a local phenomenon, boundary conditions on the whole surface of the cavity walls must be achieved through multiple interaction between all cavity sections. Therefore, multiple iterations should be carried out if a higher order solution is requested.

A detailed description of the entire process is as follows.

\section{A. Forward Propagation}

First of all, the field components that will be propagated throughout the algorithm, namely $E_{z}$ and $H_{z}$ for the $T M^{z}$ and $T E^{z}$ cases, must be sampled over the aperture. Local boundary conditions must be applied to the fields prior to the propagation through the first section. A symmetrization procedure related to classical image theory, specular for the $T E^{z}$ polarization and antispecular for the $T M^{z}$, is then applied with respect to both conductors, leading to periodic field distributions (Fig. 2). Samples over the conductors are set to zero in the $T M^{z}$ case (E-field parallel to conducting walls must vanish) but not in the $T E^{z}$ (H-Field parallel to conducting walls must correspond to a maximum or minimum).


(a)
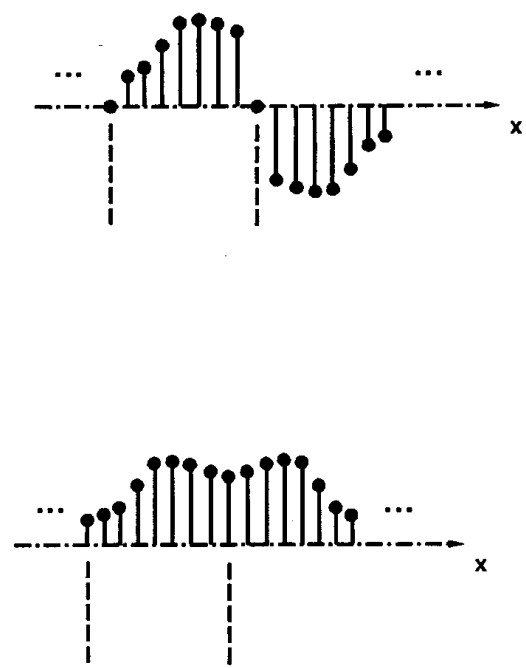

(b)

Fig. 2. Sampling and symmetrization procedure in the $T M^{z}$ (top) and $T E^{z}$ (bottom) cases. (a) Original field sampling. (b) Periodic result of applying image theory, which is processed by FFT.

The plane-wave spectrum of the resulting fields is obtained via FFT (1) and is propagated in a single step through the current waveguide section according to (2):

$$
\begin{gathered}
\tilde{U}_{z}\left(k_{x} ; y_{0}\right)=\int U_{z}\left(x, y_{0}\right) e^{j k_{x} x} d x \\
\tilde{U}_{z}\left(k_{x} ; y_{1}\right)=\tilde{U}_{z}\left(k_{x} ; y_{0}\right) e^{-j \sqrt{k^{2}-k_{x}^{2}}\left(y_{1}-y_{0}\right)}
\end{gathered}
$$

where $U_{z}=E_{z}(x, y)$ or $H_{z}(x, y)$.

The fields in the spatial domain, recovered via IFFT on the final plane (3), 


$$
U_{z}\left(x, y_{1}\right)=\frac{1}{2 \pi} \int \tilde{U}_{z}\left(k_{x} ; y_{1}\right) e^{-j k_{x} x} d k_{x}
$$

include the Huygens radiation of the fields incident to the present waveguide section plus the forward contribution of the equivalent currents along the conducting walls forming the section.

Notice that specular or antispecular symmetry is maintained after the propagation with independence of the section dimensions. Null or max-min samples on both PEC walls are also maintained after propagation.

Radiation of the equivalent currents obtained by image theory corresponds to convolution of the incident fields with the Green's function of two perfectly conducting infinite parallel planes. This approach is locally correct inside the current waveguide section. As a result, the scheme is valid only locally, so it is necessary to propagate the fields several times in forward and backward directions to take into account the multiple interaction between different waveguide sections. A global solution for the whole cavity is thus obtained, as will be seen later. As a major drawback, boundary conditions are restricted to perfect electric or magnetic conductors (PEC or PMC) so that dielectric loadings cannot be considered.

In order to adjust the sampling to waveguide sections of different widths, keeping samples over both conducting walls, an interpolation process must be carried out along the boundary between consecutive sections. Reflected field samples are to be stored if the following section is narrower than the previous one, while additional samples are introduced and set equal to zero if it is wider. In the new section, symmetrization is again applied to guarantee the fulfillment of local boundary conditions. This procedure is repeated for every waveguide section, as described in Fig. 3(a), where reflected fields are highlighted in black.

\section{B. Backward Propagation}

Once the cavity end is reached, a backward propagation procedure starts with the fields reflected in the termination endplate (Fig. 3(b)). The process is analogous to that described in Section II-A, though additional samples are not padded with zeros but with field values previously stored in the forward propagation. If further iterations are to be carried out, new reflected fields must be saved again, as indicated in Fig. 3(b).

\section{Multiple Iterations}

A first-order solution for the outwardly travelling fields is obtained after the initial iteration (forward + backward). Radiation of all interior induced currents has been taken into account, though these currents have been obtained taking into account only first-order interaction between every pair of waveguide sections. An improved solution for the currents and the scattered fields, including higher order effects between different parts of the cavity, may be obtained by additional forward + backward iterations. The scheme, for every new iteration, is analogous to that of the first one, though in the additional forward propagations there is no incident field. The only contribution to the propagating wavefront is due to the

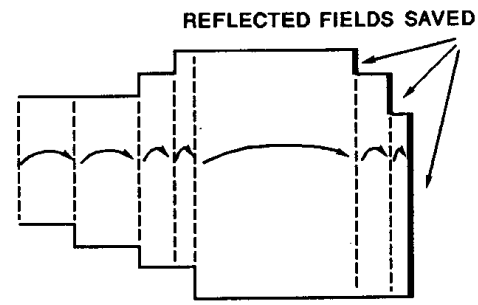

(a)



(b)

Fig. 3. Forward-backward iteration in an arbitrary 2-D cavity modeled with seven waveguide sections: (a) forward propagation and (b) backward propagation. In (a) the field reflected in wide to narrow transitions is saved to be used in the backward propagation, while in (b) the field reflected is saved to be used in the next iteration.

reflected fields stored in the previous backward iteration, which would represent higher order terms in a series expansion of the currents. The outwardly traveling fields obtained at each iteration must be coherently added over the aperture to form a higher order solution. As the amplitude of the successive wavefronts decreases constantly, a progressive convergence of the solution is achieved. Finally, the RCS can be calculated by an aperture integration based again on the Kirchhoff approximation, with some kind of edge correction if necessary.

In theory, this iterative procedure should always converge for the following reason: The fields added by each new iteration take into account a higher degree of multiple interaction between any pair of waveguide sections. In fact, the first iteration (only forward and backward propagations) computes the scattering due to the incident field and the first interaction between any pair of waveguide sections, the second forward + backward iteration adds the effect of the second-order interaction, etc. As the scattered field is the infinite summation of all these multiple interactions, and is finite, in theory the procedure should always converge. In our experience the procedure has always converged for a large number of conducting cavity configurations.

\section{Results For Two-DIMENSIONAL CAVITIES}

To illustrate the performance of the algorithm and the convergence of the interior field distribution towards a correct solution, graphical representation of the fields inside a simple cavity (Fig. 4) will be presented. The results correspond to frontal $T M^{z}$ incidence. Results for the total $E_{z}$ field along the cavity axis are shown in Fig. 4 for 1 and 12 forward-backward iterations. Notice that the solution is similar to a stationary 




Fig. 4. Field inside a 2-D simple cavity ( $y$-axis). Frontal $T M^{z}$ incidence. Magnitude of the total $E^{z}$ field after 1 and 12 iterations. Convergence is achieved in six iterations.

wave inside a transmission line. Significant improvement over the first iteration is achieved when multiple interactions are taken into account, which shows the important role played by the high-order effects at intermediate frequencies and the proper convergence of the solution. In this example, convergence is achieved in six iterations.

HF backscattering patterns of a short double-bend S-shaped cavity for the $T M^{z}$ and $T E^{z}$ polarizations are shown in Figs. 5 and 6 , where comparison has been established with reference solutions. Excellent agreement is observed with the GB shooting method and hybrid modal results [6], with a single iteration, in both $T M^{z}$ and $T E^{z}$ cases. Very slight variation is appreciated in the two-iteration results because, as previously stated, diffraction becomes a local phenomenon as the frequency is increased and, therefore, multiple interactions can be neglected.

Good agreement with GB and hybrid modal solutions [6] was also obtained for a long double-bend S-shaped cavity, as shown in Fig. 7. However, results are not so accurate at the large-angle directions because the great number of waveguide sections required to properly model such a large and complex geometry results in a certain staircase modeling error. This is particularly significant at the large aspect angles of the pattern due to the large number of ray bounces, since each ray bounce introduces a slight staircase modeling error. In addition, external edge diffraction is not included in the spectral results.

Finally, several RCS patterns for a $30^{\circ}$-offset bend cavity [8] (Fig. 8) are presented in Fig. 9. Results obtained with different cavity models and number of iterations have been checked.

\section{EXTENSION TO THREE-DIMENSIONAL CAVITIES}

Modeling procedure for nonuniform rectangular cross- section geometries (Fig. 10) is analogous to that previously described for 2-D cavities. The cavity is converted into successive straight waveguide sections. Boundary conditions are forced again via specular or antispecular images combined with FFT 2-D periodicity properties. Now a double symmetrization in both the $X$ and $Y$ directions is to be performed.

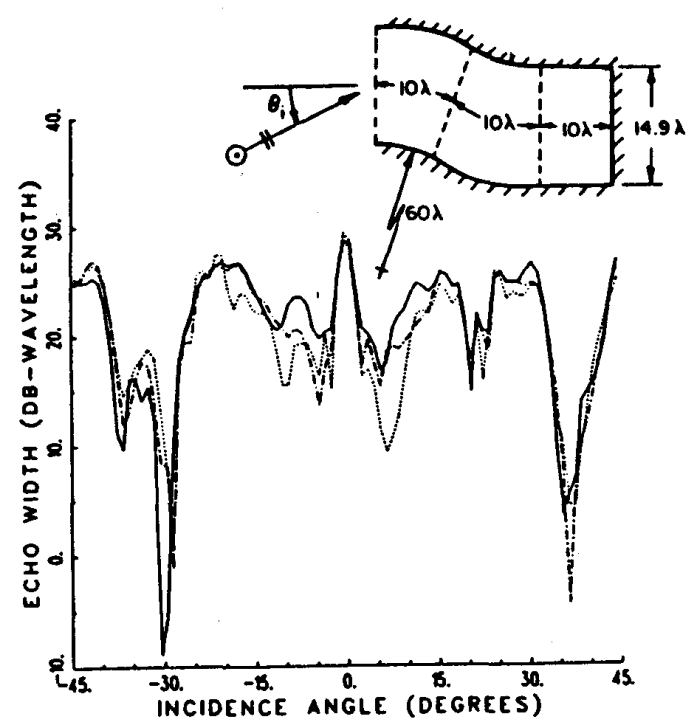

(a)

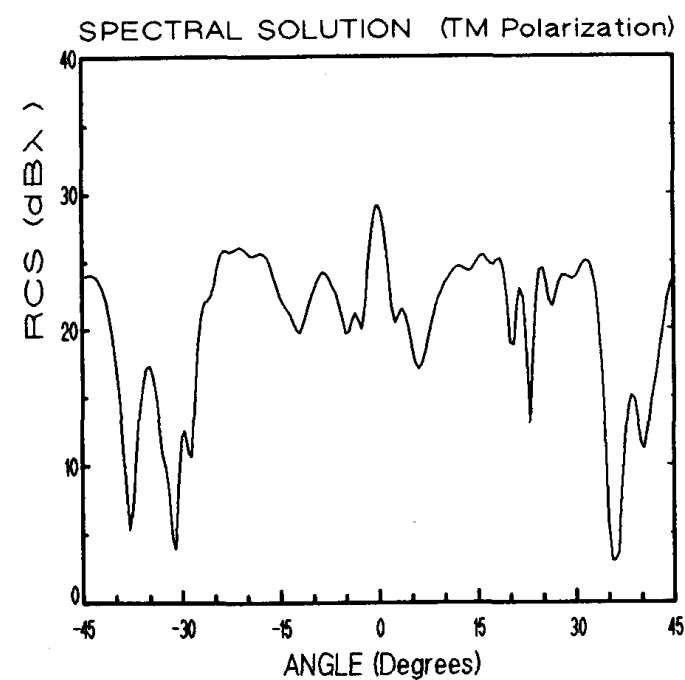

(b)

Fig. 5. RCS patterns of a short double-bend S-shaped cavity. $T M^{z}$ polarization. (a) method [6]. (b) Spectral solution, cavity modeled with 22 sections, one iteration.

Plane-wave spectrums are obtained and propagated as follows:

$$
\begin{aligned}
& \tilde{U}\left(k_{x}, k_{y} ; z_{0}\right)=\iint U\left(x, y, z_{0}\right) e^{j\left(k_{x} x+k_{y} y\right)} d x d y \\
& \tilde{U}\left(k_{x}, k_{y} ; z_{1}\right)=\tilde{U}\left(k_{x}, k_{y} ; z_{0}\right) e^{-j \sqrt{k^{2}-k_{x}^{2}-k_{y}^{2}}\left(z_{1}-z_{0}\right)} \\
& U\left(x, y, z_{1}\right)=\frac{1}{(2 \pi)^{2}} \iint \tilde{U}\left(k_{x}, k_{y} ; z_{1}\right) e^{-j\left(k_{x} x+k_{y} y\right)} d k_{x} d k_{y}
\end{aligned}
$$

where $U(x, y, z)=$ any $\vec{E}$ or $\vec{H}$ component. 


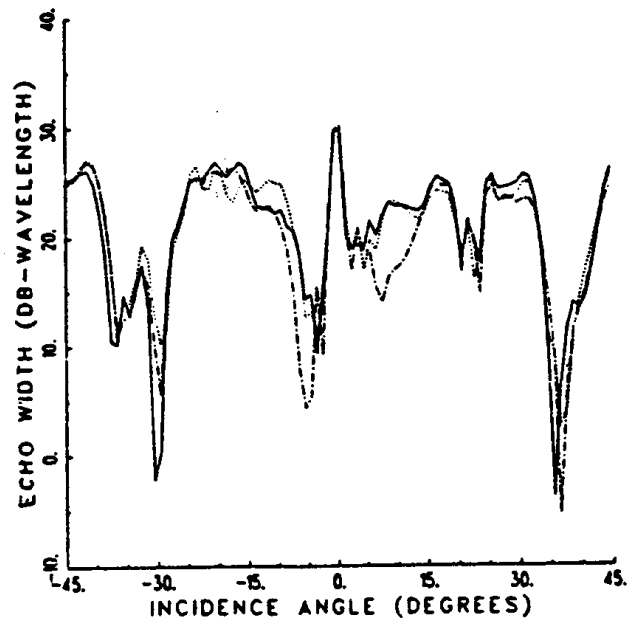

(a)

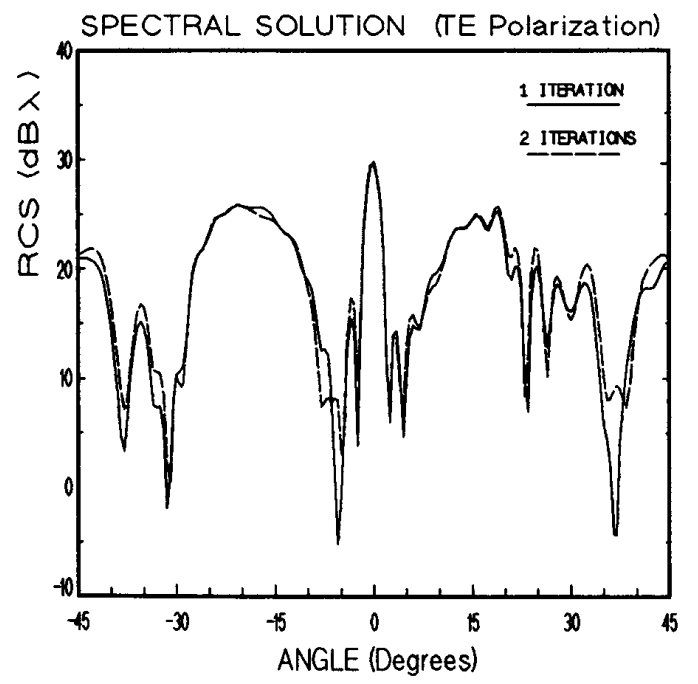

(b)

Fig. 6. RCS patterns of a short double-bend S-shaped cavity. $T E^{z}$ polarization. (a) —-: hybrid-modal reference; $\cdots \cdots,-$ - $_{-}-$: GB shooting method [6]. (b) Spectral solutions with one or two iterations, cavity modeled with 22 sections,

Given that only two of the six vectorial components of the fields are linearly independent, only two different matrixes must be used by the 3-D algorithm. The field components chosen for propagation are $E_{x}$ and $E_{y}$, which means that $T M^{z}$ and $T E^{z}$ modes are not propagated separately.

Since 2-D FFT is used, which is meaningful only in Cartesian coordinates, and since image theory is only applicable in two dimensions to rectangular cross sections with conducting walls, this procedure is applicable only to 3-D cavities with rectangular cross section.

\section{RESULTS FOR THREE-DIMENSIONAL CAVITIES}

Shown in Fig. 11(b) is the comparison between numerical results generated by the spectral algorithm and the hybrid

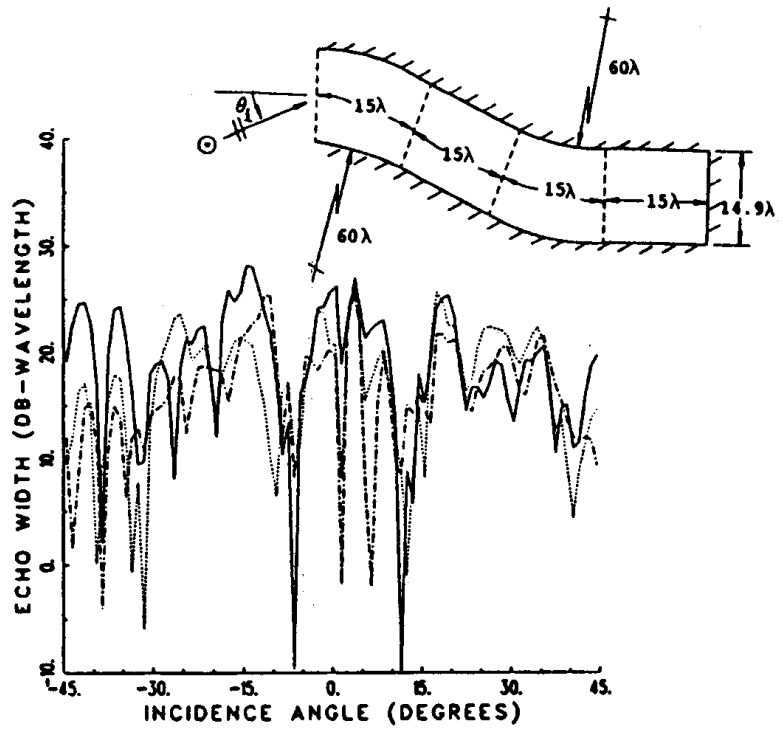

(a)

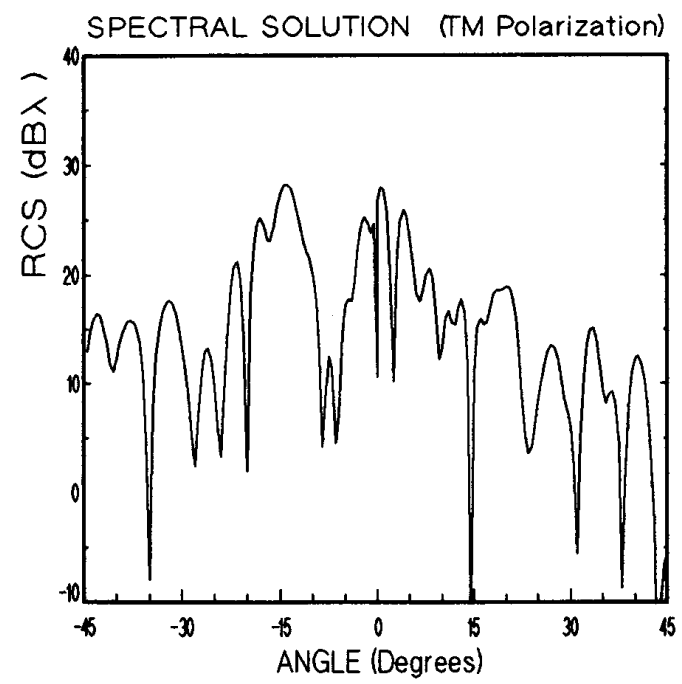

(b)

Fig. 7. RCS patterns of a long double-bend S-shaped cavity. $T M^{z}$ polarization. (a) $\longrightarrow$ : hybrid-modal reference; $\cdots \cdots,----$ : GB shooting method [6]. (b) Spectral solution, cavity modeled with 30 sections, one iteration.

BIM/modal approach, together with experimental measurements [8], for an offset rectangular waveguide cavity with two similar transition bends (Fig. 11(a)) at $10 \mathrm{GHz}$. Since this. is a difficult case for the staircase approximation, the agreement is not very good even at small aspect angles. At larger angles, additional diffraction effects and exterior scattering come into play.

A tapered waveguide cavity (Fig. 12) composed of a sectorial section with an open end connected to an uniform section with a planar termination has also been analyzed. Results at 


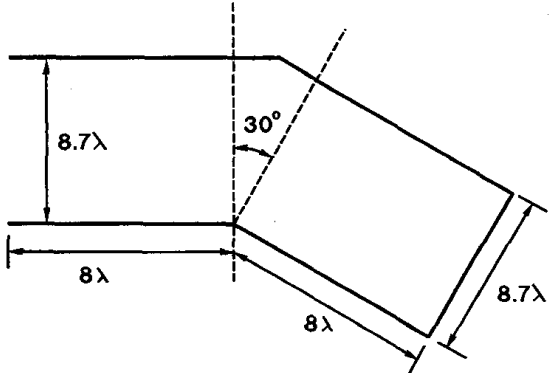

Fig. 8. Geometry of a $30^{\circ}$-offset bend cavity.

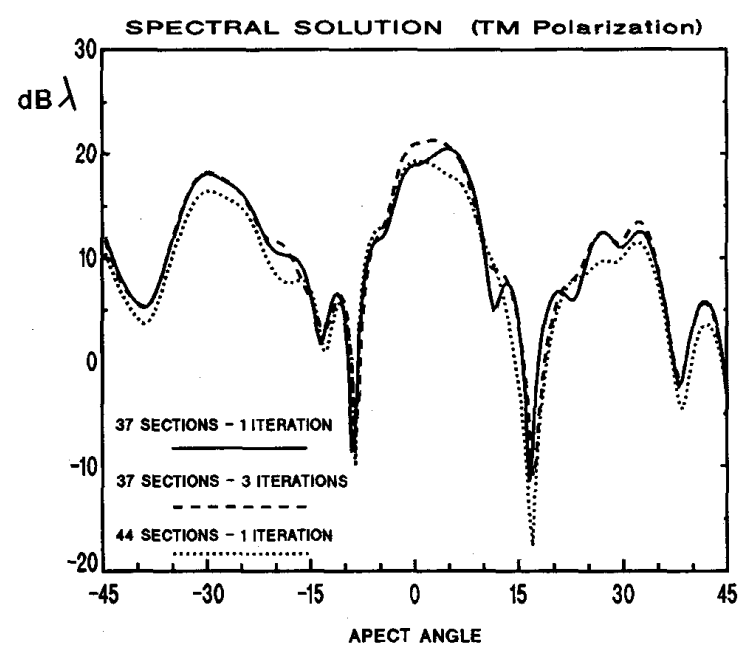

Fig. 9. Comparison between spectral solutions with different cavity segmentations ( 37 and 44 sections), $T M^{z}$ polarization.

$10 \mathrm{Ghz}$ are shown in Fig. 13 against measured RCS and modal patterns [4] for the scans corresponding to $\phi=45^{\circ}$ and $\phi=90^{\circ}$ planes. The agreement is very good for $\phi=90^{\circ}$ $\theta<60^{\circ}$ but, on the other hand, the spectral results are not valid for $\phi=45^{\circ} \theta>25^{\circ}$. The reason for that behavior is still under investigation by the authors.

\section{Discussion AND CONCLUSIONS}

The spectral iterative algorithm presented in this paper has shown remarkable features as well as some important drawbacks. To sum up, the following advantages can be pointed out:

1) The algorithm requires 1-D vectors in 2-D problems and 2-D matrixes in 3-D problems, leading to reduced storage and CPU necessities.

2) Geometrical uniformity of the cavity under analysis is exploited: In simple bodies the number of sections required is small, whereas in complex bodies many sections must be used in the stepped model. Hence, computational cost depends basically on the geometrical complexity but not on the electrical depth.

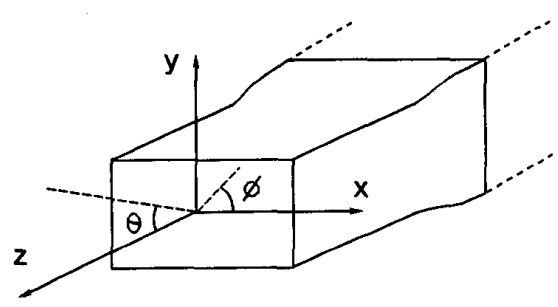

(a)

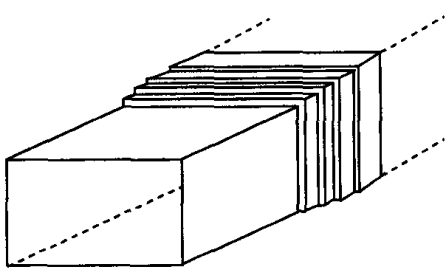

(b)

Fig. 10. Modeling procedure in a 3-D cavity with rectangular cross section. (a) Original cavity. (b) Stepped-waveguide model.

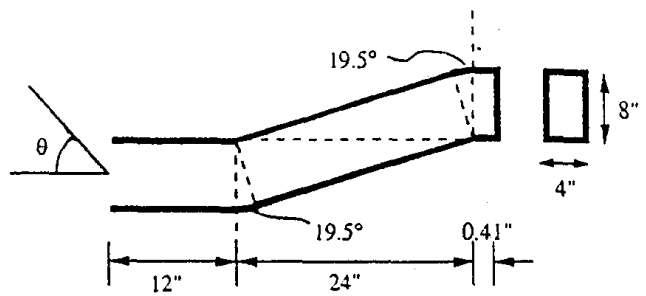

(a)

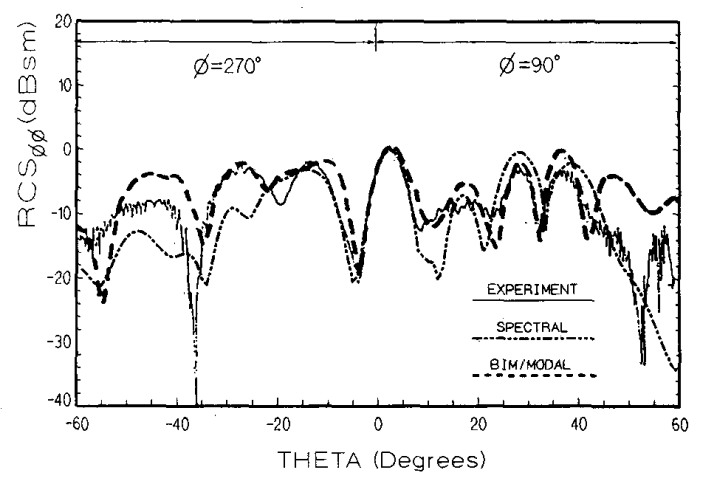

(b)

Fig. 11. (a) Geometry of the offset rectangular cavity. (b) Comparison between $\mathrm{BIM} /$ modal, experiment [8] and spectral results (two iterations, cavity modeled with 30 sections), $f=10 \mathrm{GHz}, \phi \phi$-polarization.

3) The algorithm is iterative with successive refinement. The $n$th forward-backward iteration appends to the result the fields caused by multiple interaction of order $(n+1)$ between waveguide sections. As HF scattering is a local phenomenon, only a single forward-backward 

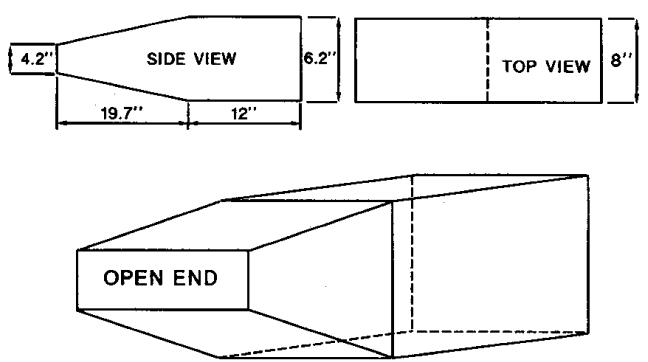

Fig. 12. Geometry of a 3-D tapered waveguide cavity.

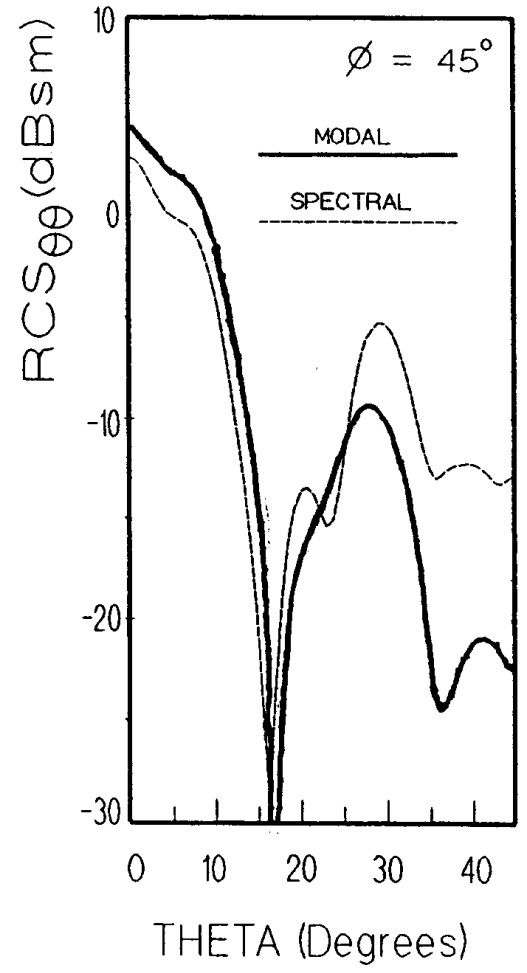

(a)

Fig. 13(a). Comparison between measured, modal [4], and spectral (two iterations, cavity modeled with 20 sections) RCS patterns, $f=10 \mathrm{GHz}$. $\theta \theta$-polarization, $\phi=45^{\circ}$ scan. (b) $\phi \phi$-polarization, $\phi=90^{\circ}$ scan.

propagation is necessary for electrically large objects, whereas a few iterations are required for scatterers of intermediate size.

As main drawbacks we should mention that:

1) The fields are distorted by the stepped shape of the cavity. In order to reduce the distortion, the modeling resolution must be increased, resulting in a higher number of sections, and thus in more FFT calculations.

2) Boundary conditions are restricted to PEC or PMC.

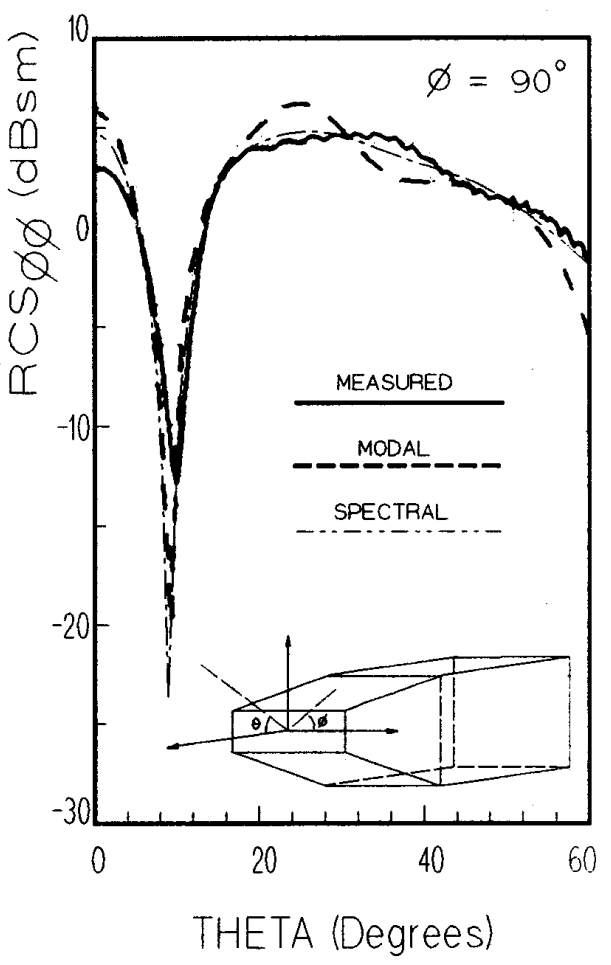

(b)

Fig. 13(b). Comparison between measured, modal [4], and spectral (two iterations, cavity modeled with 20 sections) RCS patterns, $f=10 \mathrm{GHz}$. $\phi \phi$-polarization, $\phi=90^{\circ}$ scan.

3) As stated in Section IV, the algorithm is applicable only to three-dimensional cavities with rectangular cross section, which can be modeled by successive rectangular waveguide sections.

The results provided by the new algorithm are good for a broad variety of 2-D and 3-D problems. Cavities with different shape and electrical size have been compared with numerical results reported by other authors and with experimental data, showing good agreement in the incident angles where interior backscattering is the dominant contribution to the global RCS.

The computational cost relies mainly on three parameters, which are closely interrelated:

1) Number of sections used in the stepped model of the cavity, which depends on the geometrical complexity of the original geometry.

2) Number of forward-backward iterations, which decreases with higher frequency. For electrically large cavities, a single forward-backward iteration might be enough.

3) Sampling rate. The minimum number of samples increases with the electrical size of the cross section of the duct (Nyquist rate). In addition, a certain oversampling factor over the Nyquist rate must be used so as to broaden the spectral window to account for some of the slower evanescent modes. The higher the sampling rate, 
the larger the dimension of the matrixes whose FFT has to be computed. Values ranging from 64 to 512 have been utilized in the results presented in this paper.

In conclusion, the computational cost increases with the geometrical complexity of the cavity, the number of iterations (which is usually smaller at higher frequencies) and the electrical size of the cross section of the duct.

Notice that spectral propagation is equivalent to a waveguide modal matching approach where the modes on each waveguide section are computed in a very efficient way. No more than a few seconds per incident angle in the 2-D cases and no more than a few minutes per incident angle in the 3-D problems were necessary to compute the RCS patterns in a HP-Apollo 720 workstation.

\section{REFERENCES}

[1] T. B. A. Senior, "Electromagnetic field penetration into a cylindrical cavity," IEEE Trans. Electromagn. Compat., vol. EMC-18, pp. 71-73. 1976.

[2] S. K. Jeng, "Aperture admittance matrix by finite element method for scattering from a cavity-backed aperture," in 1988 IEEE Antennas \& Propagat. Soc. Symp. Dig., Syracuse, NY, pp. 1134-1137, June 1988.

[3] H. Ling, S. W. Lee, R. Chou, "High-frequency RCS of open cavities with rectangular and circular cross sections," IEEE Trans. Antennas Propagat., vol. 37, pp. 648-654, May 1989.

[4] A. Altintas, P. H. Pathak, M. C. Liang, "A selective modal scheme for the analysis of EM coupling into or radiation from large openended waveguide cavities," IEEE Trans. Antennas Propagat., vol. 36, pp. 84-96, Jan. 1988.

[5] H. Ling, R. Chou, S. W. Lee, "Shooting and bouncing rays: Calculating the RCS of an arbitrarily shaped cavity," IEEE Trans. Antennas Propagat., vol. 37, pp. 194-205, Feb. 1989.

[6] R. Burkholder, P. H. Pathak, "Analysis of EM penetration into and scattering by electrically large open waveguide cavities using Gaussian beam shooting," in Proc. IEEE, vol. 79, pp. 1401-1411, Oct. 1991.

[7] P. H. Pathak and R. J. Burkholder, "Modal, ray and beam techniques for analyzing the EM scattering by open-ended waveguide cavities," IEEE Trans. Antennas Propagat., vol. 37, pp. 635-647, May 1989.

[8] H. Ling, "RCS of waveguide cavities: A hybrid boundary-integral/modala approach," IEEE Trans. Antennas Propagat. vol. 38, pp. 1413-1420, Sept. 1990.

[9] T. Wang and H. Ling, "Electromagnetic scattering from threedimensional cavities via a connection scheme," IEEE Trans. Antennas Propagat., vol. 39, pp. 1505-1513, Oct. 1991.

[10] R. Kastner and R. Mittra, "A spectral-iteration technique for analyzing scattering from arbitrary bodies, part I: Cylindrical scatterers with Ewave incidence," IEEE Trans. Antennas Propagat., vol. 31, May 1983.

[11] G. C. Cook, A. P. Anderson, and A. S. Turnbull, "Spectral incremental propagation (SIP) procedure for fast calculation of scattered fields from conducting bodies," Proc. IEE, vol. 136, pp. 34-28, Feb. 1989.

[12] S. W. Lee, "Comparison of uniform asymptotic theory and Ufimtsev's theory of EM edge difraction," IEEE Trans. Antennas Propagat., vol. 25, pp. 162-170, Mar. 1977.

[13] E. F. Knott, J. F. Shaeffer, and M. T. Tuley, Radar Cross Section. Artech House, 1985.

[14] R. G. Kouyoumjian and P. H. Pathak, "A uniform geometrical theory of diffraction for an edge in a perfectly conducting surface," Proc. IEEE, vol. 62 , pp. $1448-1461$, Nov. 1974

[15] N. N. Bojarski, "K-space formulation of the electromagnetic scattering problem," Air Force Avionics Laboratory, Wright-Paterson AFB, Tech. Rep. AFAL-TR-71-75, Mar. 1971.

[16] "K-space formulation of the electromagnetic scattering problem," Air Force Avionics Laboratory, Wright-Paterson AFB, Tech. Rep. AFAL-TR-72-271, Sept. 1972.

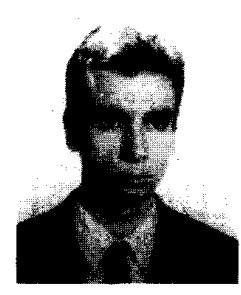

Juan M. Rius (S'89-M'92) was born in Barcelona, Spain, in 1963. He received the Ingeniero de Telecomunicacion degree in 1987 and the Doctor Ingeniero degree in 1991, both from the Universita Politécnica de Catalunya (UPC), Barcelona, Spain. In 1985 he joined the Antennas, Microwave and Radar group (AMR) at the UPC as an Undergraduate Research Assistant. Currently he is Associate Professor at the UPC. From 1985-1987 he worked on the development of cylindrical geometry algorithms for microwave tomography. Since 1988 he has been engaged in the development of the graphical processing technique (GRECO) for high-frequency RCS prediction. His current research interests involve high-frequency, spectral, and numerical techniques for electromagnetic scattering analysis.

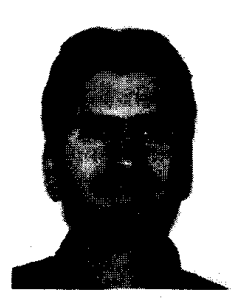

Angel Lozano was born in Manresa, Spain, in 1968 He received the Ingeniero de Telecomunicación degree in 1992 from the Universitat Politécnica de Catalunya (UPC), Barcelona, Spain. Since 1992 he has been studying for the M.S. degree in electrica engineering at Stanford University, Stanford, CA.

In the 1991-1992 period he was Undergraduate Research Assistant an the Antennas, Microwave and Radar group (AMR) at the UPC

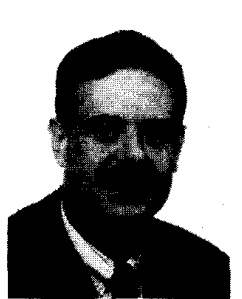

Lluis Jofre (S'79-M'83) was born in Mataro, Spain, in 1956. He received the Ingeniero de Telecomunicacion and Doctor Ingeniero degrees, both from the Universitat Politécnica de Catalunya (UPC), Barcelona, Spain, in 1978 and 1982, respectively.

In 1978 he was a Research Assistant in the Electrophysics Group at the UPC, where he worked on the analysis and near-field measurement of antennas. In 1981 he joined the Ecole Suprieure d'Electricité, Paris, France, where he was involved in microwave imaging techniques for biomedical applications. During the period 1986-87 he was Visiting Fulbright Scholar at the Georgia Institute of Technology, Atlanta, working on antenna measurements and electromagnetic imaging. He is currently Professor and Dean of the Telecommunication Engineering School, UPC, where he is engaged in research on antennas and electromagnetic scattering and imaging, both numerical and experimental aspects.

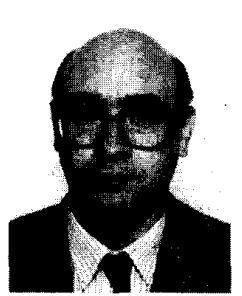

Angel Cardama (S'67-M'73) was born in Santiago, Spain, on May 13, 1944. He received the Ingeniero de Telecomunicación degree from the Universidad Politécnica de Madrid, Madrid, Spain, in 1968, and the Sc.M. and Ph.D. degrees in electrical engineering from Brown University, Providence, RI, in 1970 and 1973 , respectively.

In 1972 he joined the faculty of the Telecommunication Engineering School at the Universitat Politécnica de Catalunya, Barcelona, Spain, where he holds the position of Professor. His research interests range from propagation in optical fibers, high-frequency aperture and array antennas, and near-field antenna scanning systems to the design of microwave imaging systems and radar antennas. 